\title{
Doctors do make the worst patients
}

\author{
Sue Fferring
}

I recently had an unfortunate experience, one I am trying to erase from my memory, and writing this article is intended to be a cathartic exercise.

Having had my second baby, I made an appointment at the family planning clinic to have a Mirena ${ }^{\circledR}$ fitted 7 weeks postpartum. I was looking forward to saying goodbye to periods for a few years, and gynaecologists and family planning clinicians alike seemed to hail it as the wonder contraceptive. Having had two intrauterine devices fitted previously and, safe in the knowledge that my cervical os would be as wide as the Clyde Tunnel, fear of the procedure was not an issue. I booked my little darling into the crèche and sat and read the out-of-date glossy magazines - a far cry from the previous postnatal scenario when I had been in tears at the thought that I had abandoned my baby. This was 10 minutes of bliss.

Soon I found myself in stirrups and I felt a flutter of nerves as I reminisced to my ventouse delivery only a few weeks before. Little did I know that what was to follow would be on a par with the pain of that delivery only without the help of gas and air!

The sound went in and I felt a sharp, searing pain in my pelvis. I grimaced, stifled a moan (and then apologised) and gripped the edge of the bed. "That's the worst bit done now", I was told. Then the device itself. More of the same agonising pain followed, only this time more prolonged. I thought "should have gone for the Nova $T^{\circledR}$ " and then there was talk of spasm of the cervix, between the two doctors, followed by yet more deep, visceral pain. Then relief - all done - out came the bits and bobs followed by a bleed so dramatic it made the doctor recoil (writing this now, I wonder if it hit her clothes and I rather hope that it did). Doctor \#2 got down on her hands and knees to mop the floor and I was told not to sit up - maybe so that I maintained my blood pressure but I rather suspect it was so that I didn't see the blood.

I was washed down, given a lovely thick sanitary towel and sent on my way.

I should have known it wasn't right but somehow one doesn't want to be a wimp or a fool in front of two medical colleagues. I learnt later that was my undoing as neither of the doctors had appreciated I was in any pain even though I recall the observing doctor commenting at the time that she could see I was sore as my toes were curling! However, somehow I got home and, feeling sick with lower abdominal cramps, rifled through my stock of drug

J Fam Plann Reprod Health Care 2006; 32(2): 131

Lower Bleeding, UK

Sue Fferring, General Practitioner company handout samples and found some out-of-date Voltarol ${ }^{\circledR}$ Rapid. It did what it said on the box and I slept for a couple of hours, and thankfully so did the baby.

Less than 48 hours later I found myself in hospital awaiting a laparoscopy to fish the device out of my abdomen. I was told, "It'll be 2.30 pm" - no, now 8 pm and I was eventually taken to theatre at $10 \mathrm{pm}$. I was tired. Any 7-week postnatal woman will tell you that one 'hits the wall' at around that time and feels like a bag of raw nerves. That, coupled with more than 24 hours of fasting, left me in tatters. As the hours went by, I could imagine my dehydration affecting my lactation and I became tearful and agitated. I phoned my husband who was in the throes of toddler teatime and baby feeding and wished I could be there.

Being an inpatient on a surgical ward sucks - more so for a female doctor, or maybe I was being paranoid. When 'coming round' I knew I was uncomfortable and tried to fathom out why. When I had worked out that I had enough reasons to summon a nurse from her midnight feast at the nurses' station, I reached for my buzzer and waited. Sister arrived at my bedside and I said, "I'm cold and thirsty, that street light is shining in my eyes and I think I'm lying in a pool of blood!". She replied, "Nothing's right for you, is it?" then lifted the covers and said, "Oh dear, I'll get some clean sheets".

Since my house officer days I have had an issue with nurses, but was I left stranded (on purpose?) on that commode with no toilet paper and out of reach of a buzzer for 20 minutes when all I had asked for was a pee. The next morning, did they deliberately leave me for an hour attached to my bed by my drip after my request to use the toilet? Rather than wet the bed, I capped off the intravenous line myself. Again, in the morning, we were all asked who needed a bowl and who could get up to use the bathroom to wash and, when I replied that I could get up, the retort was "What are you waiting for, Christmas?!". I so desperately wanted to get out but was told that nobody gets discharged from that ward so I had to wait for two porters to take me in a minibus to the gynaecology ward just so that I could be released!

I think there are lessons to be learned here. First, don't tell anyone you are a doctor and then you can scream expletives loudly when you are having your uterus perforated and someone may think of doing something about it. Second, just because we are doctors we do not have to have the attitude that "I must be able to tolerate anything, Joe Public can". Most of us are human after all. Third, if you are female and happen to end up on the ward I was on, and you let it slip that you are a doctor, make sure you have your own bedpan with you.

\section{LETTERS TO THE EDITOR}

Letters to the Editor are welcome and generally should not exceed 600 words or cite more than five references. For comments on material published in the most recent issue of the Journal, correspondence should be received within 4 weeks of dispatch of that Journal to be in time for inclusion in the next issue. When submitting letters correspondents should include their job title, a maximum of two qualifications and their address(es). A statement on competing interests should also be submitted for all letters. Letters may be submitted to the Editor or the Journal Editorial Office (details on page 65). 